\title{
Alterstice
}

Revue internationale de la recherche interculturelle

International Journal of Intercultural Research

Revista International de la Investigacion Intercultural

\section{La gestion de la différence culturelle par les éducateurs sociaux dans leur travail au quotidien}

\section{Alida Gulfi}

Volume 5, numéro 2, 2015

URI : https://id.erudit.org/iderudit/1036696ar

DOI : https://doi.org/10.7202/1036696ar

Aller au sommaire du numéro

Éditeur(s)

Alterstice

ISSN

1923-919X (numérique)

Découvrir la revue

Citer cet article

Gulfi, A. (2015). La gestion de la différence culturelle par les éducateurs sociaux dans leur travail au quotidien. Alterstice, 5(2), 109-122.

https://doi.org/10.7202/1036696ar

\section{Résumé de l'article}

L'intervention en contexte multiculturel constitue un défi pour les éducateurs sociaux qui doivent composer avec la diversité culturelle. Cet article s’intéresse à la gestion de la différence culturelle par les éducateurs sociaux dans leur travail au quotidien. Quelle est la place qu'ils donnent à la différence culturelle dans l'intervention? Quels sont les défis qu'ils rencontrent dans l'accompagnement des usagers aux références culturelles diverses? Pour ce faire, une enquête qualitative a été conduite auprès de 24 éducateurs sociaux travaillant dans diverses institutions socio-éducatives de Suisse romande. Les résultats mettent en évidence trois types d'attitude qui caractérisent la gestion de la différence culturelle par les éducateurs sociaux : une même intervention pour tous les usagers quelle que soit leur origine, une intervention spécifique et individualisée pour chaque usager et une adaptation de l'intervention aux spécificités culturelles des usagers. De même, les résultats montrent que les éducateurs sociaux sont confrontés à plusieurs défis d'ordre personnel, relationnel et contextuel dans le travail avec la différence culturelle, notamment les relations de genre, la conception du handicap et de la maladie mentale, les modes d'éducation des enfants, les croyances et pratiques religieuses, les contraintes administratives, institutionnelles et politiques liées à l'intervention ainsi que les trajectoires migratoires des usagers et leurs familles. 


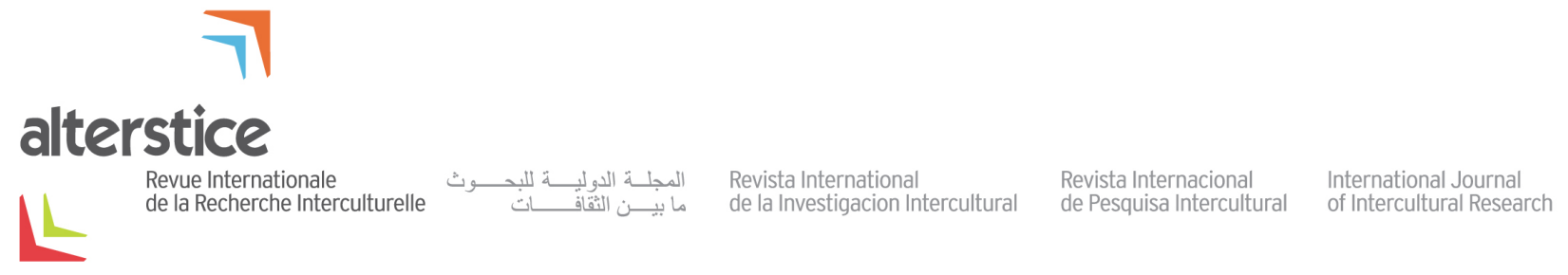

ARTICLE HORS THÈME

\title{
La gestion de la différence culturelle par les éducateurs sociaux dans leur travail au quotidien
}

\author{
Alida Gulfi ${ }^{1}$
}

\section{Résumé}

L'intervention en contexte multiculturel constitue un défi pour les éducateurs sociaux qui doivent composer avec la diversité culturelle. Cet article s'intéresse à la gestion de la différence culturelle par les éducateurs sociaux dans leur travail au quotidien. Quelle est la place qu'ils donnent à la différence culturelle dans l'intervention? Quels sont les défis qu'ils rencontrent dans l'accompagnement des usagers aux références culturelles diverses? Pour ce faire, une enquête qualitative a été conduite auprès de 24 éducateurs sociaux travaillant dans diverses institutions socioéducatives de Suisse romande. Les résultats mettent en évidence trois types d'attitude qui caractérisent la gestion de la différence culturelle par les éducateurs sociaux : une même intervention pour tous les usagers quelle que soit leur origine, une intervention spécifique et individualisée pour chaque usager et une adaptation de l'intervention aux spécificités culturelles des usagers. De même, les résultats montrent que les éducateurs sociaux sont confrontés à plusieurs défis d'ordre personnel, relationnel et contextuel dans le travail avec la différence culturelle, notamment les relations de genre, la conception du handicap et de la maladie mentale, les modes d'éducation des enfants, les croyances et pratiques religieuses, les contraintes administratives, institutionnelles et politiques liées à l'intervention ainsi que les trajectoires migratoires des usagers et leurs familles.

\section{Rattachement de l'auteure \\ ${ }^{1}$ Haute école de travail social Fribourg, Givisiez, Suisse}

\section{Correspondance}

alida.gulfi@hefr.ch

\section{Mots clés}

éducateurs sociaux, intervention, usagers, différence culturelle, gestion, défis

\section{Pour citer cet article}

Gulfi, A. (2016). La gestion de la différence culturelle par les éducateurs sociaux dans leur travail au quotidien Alterstice, 5(2), 109-122. 


\section{Introduction}

En Suisse, tout comme dans la plupart des sociétés dites globalisées, les éducateurs sociaux doivent composer avec la diversité culturelle dans leur travail. D'une part, les publics qui fréquentent les institutions socio-éducatives sont de plus en plus variés, que ce soit sur le plan culturel, linguistique ou social. Selon les études menées à ce jour, les personnes de nationalité étrangère sont en effet particulièrement exposées au risque d'être affectées par des contraintes psycho-sociales concernant notamment la condition migratoire (trajectoire migratoire, statut de séjour), la situation socio-économique (faible niveau de qualification, accès limité à l'emploi, précarité financière), la situation face à l'éducation (inégalité des opportunités, accès limité à la formation), la position sociale et le parcours d'intégration (déclassement social, participation sociale et politique difficile, influence limitée sur la répartition des biens collectifs), l'état de santé (problèmes de santé, accès limité aux soins), la discrimination, voire le racisme (Chaudet, Regamey, Rosende Haver et Tabin, 2000; Lanfranchi, 2002). Ces éléments font que les personnes de nationalité étrangère sont surreprésentées comme usagères du travail social dans le contexte sociopolitique et économique actuel (Bolzman et Tabin, 1999). D'autre part, l'hétérogénéité des références culturelles est visible également parmi les professionnels, même si la proportion d'étrangers parmi les travailleurs sociaux demeure faible (Eckmann et Bolzman, 2002) et les étudiants étrangers ne sont pas encore très présents dans le domaine du travail social (OFS, 2014). Cette diversification croissante introduit dans l'intervention des éléments nouveaux que les éducateurs sociaux sont amenés à prendre en compte, ce qui peut complexifier leur pratique habituelle. En effet, des études antérieures montrent qu'aussi enrichissant qu'il soit, le travail avec la différence culturelle implique un certain nombre de défis pour les professionnels de l'humain, qu'ils soient enseignants (Edelmann, 2007; Meijer, 2009; Ogay, 2006; Tatar et Horenczyk, 2003), travailleurs sociaux (Cohen-Emerique, 2011; Guelamine, 2001; Prieur, Jovelin et Leblanc, 2006; Steiger et Pfiffner, 2008; Verbunt, 2004) ou intervenants de la santé (Dominicé Dao et Bélanger, 2007; Fortin et Carle, 2007; Layat Burn, 2007; Leanza, 2011). Les problèmes relevés par ces professionnels sont principalement liés à la langue (Alvarado, 2004; Cohen, Rivara, Marcuse, McPhillips et Davis, 2005; Nehring, 2007; Piérart, 2013) et à la culture (représentations et pratiques divergentes) (Cohen et collab., 2005; Eckmann, Sebeledi, Bouhadouza Von Lanthen et Wicht, 2009; Kirmayer, 2011).

Les éducateurs sociaux interviennent auprès de personnes de tous âges qui présentent des difficultés variées dans un cadre institutionnel qui répond à des mandats et missions spécifiques. Dans ce cadre, la relation d'aide peut être à l'origine de relations de pouvoir inégales et de situations de tension ou de conflit entre le professionnel et l'usager. Dans l'intervention en contexte multiculturel s'y ajoutent les statuts sociaux et juridiques totalement différents entre éducateurs sociaux et usagers ainsi que le contexte social, politique, économique et historique dans lequel se déroule la relation (Bolzman, 2009). Ainsi, l'intervention interculturelle peut susciter, chez des personnes placées hors de leur contexte socioculturel (ici l'éducateur social et l'usager), des réactions émotionnelles et intellectuelles positives (fascination, enthousiasme) ou négatives (dépaysement, frustrations, rejet) qui vont orienter les comportements respectifs et, en situation professionnelle, générer des confrontations nuisibles à l'intervention (Cohen-Emerique, 2011). Certains professionnels peuvent ainsi être amenés à questionner leurs normes et modèles de pratiques, leurs rôles et pratiques professionnels, voire la culture de l'institution dans laquelle ils œuvrent (Cohen-Emerique, 2011; Leanza, 2011; Piérart, 2013). C'est dire si la problématique de l'accompagnement en contexte multiculturel est d'actualité pour les éducateurs sociaux et le restera longtemps.

Cet article porte sur l'expérience de la différence culturelle vécue par les éducateurs sociaux dans leur travail au quotidien. Comment les éducateurs sociaux intègrent-ils la différence culturelle dans l'intervention auprès des usagers? Quelles sont les différences culturelles qui, d'après les éducateurs sociaux, sont à l'origine de malentendus, d'incompréhensions ou de conflits dans l'intervention interculturelle et qui leur posent ainsi des défis dans la pratique?

\section{Une perspective interculturelle et écosystémique}

L'expérience de la différence culturelle vécue par les éducateurs sociaux dans leur travail au quotidien a été envisagée dans une vision interculturelle écosystémique (Cohen-Emerique, 2000 et 2011; Legault et Rachédi, 2008). Cette perspective prend comme point de départ les deux acteurs de l'intervention, ici l'éducateur social et l'usager. Tous deux sont porteurs de valeurs (par exemple, conception de la personne, de la famille, de l'éducation 
des enfants, de la vie en société) et d'une histoire de vie qui leur est propre, qui vont influencer leurs attitudes et actions. D'autres éléments liés à leurs appartenances respectives, tels que la profession, l'éducation, l'origine, le statut ou la religion, jouent un rôle dans l'intervention. L'ensemble de ces éléments constitue le cadre de référence de chacun, c'est-à-dire les représentations dont ils sont porteurs et à partir desquelles ils interprètent la situation d'interaction. Il s'agit de la dimension subjective de l'intervention interculturelle. Les cadres de référence respectifs constituent des filtres de l'interaction et peuvent créer des malentendus, incompréhensions et jugements de valeur. Si ces problèmes ne sont pas résolus, ils peuvent avoir des conséquences négatives sur la relation entre l'éducateur social et l'usager (dimension relationnelle). L'intervention interculturelle comprend également une dimension situationnelle liée au contexte de l'intervention qui comprend, par exemple, les missions et mandats institutionnels, les réseaux professionnels et non professionnels qui entourent l'usager, la culture professionnelle, les politiques éducatives et sociales en vigueur. Dans cette perspective, la différence culturelle est présente dans l'intervention en raison de l'interaction entre les cadres de références de l'institution et de ses principaux acteurs, les éducateurs sociaux et les usagers (Ogay et Edelmann, 2011).

\section{Méthode \\ Déroulement}

La collecte des données a été réalisée par le biais d'entretiens individuels semi-directifs (Blanchet et Gotman, 2006), en vue de porter un éclairage sur la manière dont les éducateurs sociaux prennent en compte la différence culturelle dans leur travail au quotidien. Les participants ont tout d'abord été invités à s'exprimer sur leurs attitudes face aux usagers se référant à une autre culture ${ }^{1}$. Ensuite, ils ont été invités à raconter, par le biais de la " méthode des incidents critiques » (Cohen-Emerique, 2011; Eckmann et collab., 2009; Piérart, 2013), une situation significative vécue ou observée récemment dans le travail auprès d'usagers aux références culturelles diverses et leur entourage. Les données recueillies auprès des éducateurs sociaux ont été enregistrées sur un support audio et ensuite traitées par le biais de la méthode d'analyse de contenu thématique (Bardin, 2007; Miles et Huberman, 2003). Les situations ont été analysées à partir du "modèle de l'intervention interculturelle écosystémique » (Cohen-Emerique, 2011; Legault et Rachédi, 2008).

\section{Participants}

Cette étude a été menée auprès de 24 éducateurs sociaux qui interviennent auprès d'enfants, adolescents et jeunes adultes en situation familiale, sociale, scolaire, d'apprentissage ou d'intégration difficile $(n=14)$, de personnes en situation de handicap (mental, physique ou psychique) $(n=9)$ ou d'adultes présentant des problèmes de dépendance $(n=1)$ dans diverses institutions socio-éducatives en Suisse romande. Conformément à la féminisation des professions du domaine social, plus de femmes ( $n=15)$ que d'hommes $(n=9)$ ont pris part à l'enquête. Leur âge moyen est de 32 ans (étendue allant de 27 à 47 ans), 15 étant âgés de 30 ans au plus. La majorité des participants sont suisses $(n=17), 5$ ont la double nationalité suisse et étrangère et 2 sont étrangers mais vivent en Suisse depuis plusieurs années. Environ la moitié des participants $(n=13)$ a vécu dans une autre région linguistique de Suisse ou dans un autre pays pour une période d'au moins trois mois, en raison du travail, des études ou encore de l'envie de découvrir d'autres régions/pays ou cultures. Le tableau 1 présente les principales caractéristiques sociodémographiques et professionnelles des participants.

\footnotetext{
${ }^{1}$ Le terme personnes aux références culturelles diverses est utilisé dans cet article pour prendre en compte à la fois l'aspect pluriel, dynamique, évolutif, "individualisé » et relationnel de la culture, comme le soulignent Abdallah-Pretceille (1985) et Camilleri (1989) : I'individu n'a pas une culture, mais il y fait référence pour construire son rapport à des significations culturelles en fonction de son histoire personnelle, des contextes et des interactions avec autrui.
} 
Tableau 1 : Caractéristiques sociodémographiques et professionnelles des 24 participants

\begin{tabular}{|c|c|c|c|c|}
\hline & Genre & $\begin{array}{l}\text { Âge } \\
\text { (ans) }\end{array}$ & Nationalité & Milieu de pratique \\
\hline ES1 & $\mathrm{F}$ & 28 & Suisse & Foyer pour personnes en situation de handicap ${ }^{a}$ \\
\hline ES2 & $\mathrm{H}$ & 40 & Suisse & Institution pour jeunes en difficulté ${ }^{b}$ \\
\hline ES3 & $\mathrm{F}$ & 30 & Suisse & Foyer pour personnes en situation de handicap \\
\hline ES4 & $\mathrm{F}$ & 27 & Binationale & Foyer pour personnes en situation de handicap \\
\hline ES5 & $\mathrm{H}$ & 31 & Suisse & Centre de jeunesse \\
\hline ES6 & $\mathrm{F}$ & 27 & Suisse & Institution pour jeunes en difficulté \\
\hline ES7 & $\mathrm{F}$ & 28 & Suisse & Institution pour jeunes en difficulté \\
\hline ES8 & $\mathrm{H}$ & 30 & Binational & Institution pour jeunes en difficulté \\
\hline ES9 & $\mathrm{F}$ & 30 & Étrangère & Institution pour jeunes en difficulté \\
\hline ES10 & $\mathrm{H}$ & 44 & Binational & Institution pour jeunes en difficulté \\
\hline ES11 & H & 37 & Suisse & $\begin{array}{l}\text { Structure pour parents en difficulté dans l'éducation de } \\
\text { leurs enfants }\end{array}$ \\
\hline ES12 & $\mathrm{F}$ & 47 & Suisse & Institution pour jeunes en difficulté \\
\hline ES13 & $\mathrm{H}$ & 30 & Suisse & Centre pédagogique pour jeunes en situation de handicap \\
\hline ES14 & $\mathrm{F}$ & 30 & Suisse & Foyer pour personnes en situation de handicap \\
\hline ES15 & $\mathrm{H}$ & 35 & Suisse & Centre de formation professionnelle et sociale \\
\hline ES16 & $\mathrm{F}$ & 30 & Binationale & Foyer pour personnes en situation de handicap \\
\hline ES17 & $\mathrm{H}$ & 41 & Suisse & Structure pour adultes avec des problèmes psychosociaux \\
\hline ES18 & $\mathrm{F}$ & 35 & Étrangère & Foyer pour personnes en situation de handicap \\
\hline ES19 & $\mathrm{F}$ & 30 & Suisse & Foyer pour personnes en situation de handicap \\
\hline ES20 & $\mathrm{H}$ & 29 & Suisse & Institution pour jeunes en difficulté \\
\hline ES21 & $\mathrm{F}$ & 29 & Binationale & Centre d'animation pour la jeunesse \\
\hline ES22 & $\mathrm{F}$ & 27 & Suisse & Institution pour jeunes en difficulté \\
\hline ES23 & $\mathrm{F}$ & 33 & Suisse & $\begin{array}{c}\text { Centre pour personnes avec des problèmes de } \\
\text { dépendance }\end{array}$ \\
\hline ES24 & $\mathrm{F}$ & 29 & Suisse & École spécialisée \\
\hline
\end{tabular}

Abréviations: $\mathrm{F}$, femme; $\mathrm{H}$, homme; milieu de pratique in extenso: ${ }^{a}$ home ou foyer pour personnes en situation de handicap (mental, physique, psychique); ${ }^{b}$ Foyer ou structure de jour pour enfants, adolescents et jeunes adultes en difficulté (familiale, sociale, scolaire, d'apprentissage, d'intégration)

\section{Résultats}

Les attitudes des éducateurs sociaux face aux usagers aux références culturelles diverses

Les participants ont d'abord été invités à exposer la façon dont ils prennent en compte les références culturelles des usagers dans l'intervention. Des témoignages, il ressort que les attitudes qui caractérisent l'accompagnement des usagers aux références culturelles diverses par les éducateurs sociaux se déclinent sous trois formes: une même intervention pour tous, une intervention spécifique à la situation de chacun et une adaptation de l'intervention aux besoins et spécificités des personnes aux références culturelles diverses (Leanza, 2011; Steiger et Pfiffner, 2008). 
Le premier type d'attitude que l'on rencontre chez les éducateurs sociaux, c'est la volonté d'intervenir de la même manière auprès de tous les usagers, quelle que soit leur origine culturelle.

On travaille de la même manière, ça ne change pas... (ES1, femme, 28 ans)

Non, je n'ai pas l'impression que ça se passe différemment. (ES15, homme, 35 ans)

Cette attitude, qui est fréquente chez les professionnels de l'humain, fait écho aux valeurs humanistes, démocratiques et universalistes qui sous-tendent l'éducation sociale, notamment le principe d'égalité de traitement et de respect pour tous les usagers, le refus de toute forme de discrimination ainsi que le devoir de reconnaître la différence (Avenir Social, 2010). Toutefois, la minimisation, voire la négation des différences culturelles soulève de plus en plus de questionnements dans l'intervention, car les éducateurs sociaux risquent, d'une part, de marginaliser et discriminer des publics porteurs de références culturelles ou ethniques spécifiques (Gianni, 2005), d'autre part, de ne pas répondre de manière adéquate à leurs difficultés et besoins spécifiques (Ogay et Edelmann, 2011).

Le deuxième type d'attitude consiste, pour les éducateurs sociaux, à intervenir auprès de chaque usager de manière spécifique et individualisée. Ils estiment que chaque usager est unique et possède ses spécificités au niveau des valeurs, de la personnalité, de l'histoire de vie ou encore du cadre de référence.

Chaque résidant a des particularités et des choses spécifiques et puis je pense que chacun a ses particularités et ce n'est pas forcément une histoire de culture. (ES3, femme, 30 ans)

La position des éducateurs sociaux est de maximiser les différences individuelles pour minimiser celles dites culturelles. Aux yeux des éducateurs sociaux, l'origine culturelle de l'usager doit être prise en compte comme un facteur parmi d'autres et l'éducateur social doit s'intéresser, outre à sa problématique, à tous les éléments du cadre de référence (personnalité, comportement, âge, histoire de vie) de l'usager.

En fait, on fait de l'accompagnement individualisé, donc peu importe la culture finalement. Quand on rencontre la personne, ça va être à voir s'il demande quelque chose, s'il a besoin d'un coup de pouce ou pas, comment ça marche dans la relation, comment il fonctionne aussi et puis c'est individualisé, mais pas spécialement en fonction de la culture... beh de toute manière, comme un autre facteur en fait... (ES21, femme, 29 ans)

Cette attitude, qui met l'accent sur les différences interindividuelles comme facteur explicatif majeur, s'inscrit dans une logique individuo-universaliste (Leanza, 2011). Le risque est que les différences et les difficultés rencontrées par les usagers aux références culturelles diverses soient personnalisées et individualisées sans que les éducateurs sociaux prennent en compte la dimension collective d'une discrimination ou d'une position particulière de ces personnes (Eckmann, 2004).

Le troisième et dernier type d'attitude que l'on observe est l'intégration, par les éducateurs sociaux, de la dimension interculturelle dans l'intervention au moyen d'ajustements spécifiques dans l'accompagnement des personnes aux références culturelles diverses.

Oui j'essaie, mais ce n'est pas toujours évident parce qu'on n'a pas toujours la connaissance nécessaire pour le faire. La connaissance de la perception de l'autre donc il y a tout un travail de... qui consiste déjà à imaginer qu'il y a plein de perceptions possibles pour une même situation donnée, chacun la perçoit d'une autre manière, et puis essayer de se mettre à la place de l'autre. (ES18, femme, 35 ans)

Cette lecture traduit une représentation ethnoculturelle de l'intervention (Bolzman, 2009) : les éducateurs sociaux prennent en compte les spécificités culturelles des usagers pour mieux comprendre leur problématique et éviter des pistes interprétatives basées uniquement sur les modèles et pratiques professionnels ainsi que sur les missions et mandats institutionnels. Bien qu'étant basé sur une idée très positive de la différence, le souhait d'adapter l'intervention en fonction des références culturelles des usagers pourrait mener les éducateurs sociaux à réduire ces personnes à leurs seules références culturelles et à les stigmatiser (Ogay et Edelmann, 2011). 
Ces différentes attitudes traduisent la tension qu'éprouvent les éducateurs sociaux quant à la manière de considérer la différence culturelle dans l'intervention. Doivent-ils minimiser, voire nier les différences culturelles afin de garantir l'égalité de traitement à tous les usagers et éviter ainsi toute forme de discrimination? Ou mettre l'accent sur les différences interindividuelles afin de personnaliser et individualiser toute intervention? Ou encore reconnaître et adapter l'intervention en fonction des différences culturelles? En ce sens, la gestion de la différence culturelle consiste, pour les éducateurs sociaux, à trouver le juste équilibre entre l'égalité et la différence, en adaptant l'intervention en fonction de la problématique et du cadre de référence des usagers, des missions et mandats institutionnels, tout en vérifiant que les usagers ne sont pas victimes de discrimination et de traitement inégal en raison de leurs références culturelles.

Finalement, les résultats indiquent qu'aucun parallélisme ne peut être tiré entre les attitudes des éducateurs sociaux face aux usagers aux références culturelles diverses et le milieu de pratique dans lequel ils exercent.

\section{Les défis rencontrés par les éducateurs sociaux dans le travail avec la différence culturelle}

Les participants ont également été invités à raconter une situation professionnelle vécue ou observée récemment avec une personne aux références culturelles diverses qui les avait rendus particulièrement incertains ou qui leur avait posé question.

Les situations problématiques rapportées par les éducateurs sociaux peuvent être regroupées en six grandes catégories : (1) la vision des statuts et des rôles des femmes et des hommes, (2) la conception du handicap et de la maladie, (3) l'éducation des enfants, (4) les croyances et pratiques religieuses, (5) l'intervention et le contexte institutionnel ainsi que (6) les trajectoires migratoires des usagers.

La vision des statuts et des rôles des femmes et des hommes

La première catégorie, qui nous a été rapportée par environ la moitié des éducateurs sociaux tous milieux de pratique confondus, concerne la perception différenciée des statuts et des rôles des femmes et des hommes dans la famille et dans la société ainsi que les relations de genre.

La conception de la famille, ainsi que celle des statuts et des rôles de ses membres (c'est-à-dire père, mère, fils et fille) en son sein en fonction du genre, diffèrent d'une communauté culturelle à l'autre. Or les éducateurs sociaux qui interviennent auprès d'usagers aux références culturelles diverses se trouvent parfois confrontés à une organisation ou un fonctionnement familial qui ne leur sont pas familiers. Au sein de certaines familles, les statuts et les rôles sont différenciés et complémentaires en fonction du genre. Dans ces situations, les éducateurs sociaux, qui se situent généralement dans une perspective d'égalité des genres, voient dans les rapports entre hommes et femmes une relation où les hommes exercent une autorité despotique sur les femmes, qui de leur côté font preuve de soumission et de dépendance, comme le montre l'extrait ci-dessous.

Il y a des moments aussi qui sont durs, qu'il peut répondre aussi à sa femme "Tais-toi, tu ne sais rien, tout ça ». Là, deux ou trois fois j'ai dû avaler ma salive parce que "gloups ». [...] J'ai juste... ça c'est quelque chose qu'on remarque... le regard qu'elle lance vers son mari, si elle a le droit de dire ou si elle n'a pas le droit de dire et puis de toute façon elle est effacée, de toute façon. (ES18, femme, 35 ans)

Cette vision différenciée des relations de genre, et plus spécifiquement du statut et du rôle de la femme dans la famille, peut entraver la collaboration entre les éducateurs sociaux et les familles aux références culturelles diverses (Vatz Laaroussi, 2008).

Un autre cas de figure est constitué par les rapports hommes/femmes dans la société. Les éducatrices sociales se plaignent des attitudes et comportements dénigrants, voire machistes, de quelques usagers masculins ou pères de famille à leur encontre, en mettant en avant comme facteur explicatif la religion ou la culture.

Oui, et bien par exemple aussi avec des personnes qui viennent des Balkans, dans certaines cultures la femme n'est pas tellement perçue comme une personne, comment dire... importante. Enfin, elle est en tout cas moins importante que 
l'homme donc aussi essayer de leur faire comprendre ça, qu'ici ce n'est pas pareil, qu'ils ont aussi à respecter les éducatrices qui sont femmes, autant que les éducateurs hommes. (ES7, femme, 28 ans)

Et puis où le père [musulman] ne regardait même pas l'éducatrice, il parlait avec... parce que c'était une femme, ou bien qu'il la prenait pour une moins que rien. (ES10, homme, 44 ans)

Ces situations liées aux relations de genre créent du malaise et des tensions, notamment chez les éducatrices sociales, qui essaient de se faire respecter en tant que professionnelles et femmes.

Et puis avec les personnes d'une autre culture... ça a été difficile avec certains résidents, un monsieur arabe en l'occurrence, qui avait une image des femmes très négative. [...] Ça je trouvais dur, d'être professionnelle, d'être... oui, de rester dans son rôle professionnel quand on sait que l'autre personne a une image négative ou même dénigrante de la femme. (ES14, femme, 30 ans)

Toutefois, comme on l'a déjà mentionné, la relation d'aide est caractérisée par la position asymétrique dans laquelle se trouvent l'éducateur social et l'usager ou ses proches. Dès lors, ces situations peuvent traduire davantage des difficultés liées aux rapports de pouvoir inégaux dans l'intervention que des perceptions différenciées des relations de genre (Eckmann et collab., 2009).

Finalement, deux éducatrices sociales dénoncent des relations conflictuelles basées sur des stéréotypes de genre entre collègues ou avec des supérieurs hiérarchiques.

ES1 (femme, 28 ans) a entendu son directeur maghrébin dire à une collègue « Écoutez, t’es une femme donc... là, entre guillemets tu te tais quoi $»$.

Ces situations contreviennent au principe de la non-discrimination visant à assurer l'égalité de traitement dans les rapports de travail, tel qu'il est énoncé dans la Loi fédérale sur l'égalité entre femmes et hommes (LEg, 2011).

\section{La conception du handicap et de la maladie}

Une autre catégorie de situations problématiques relevées par les éducateurs sociaux concerne la compréhension différenciée du handicap et de la maladie mentale, notamment du diagnostic, de l'étiologie et de la symptomatologie, ainsi que de l'intervention socio-éducative et thérapeutique. En toute logique, cette catégorie de situations a été évoquée uniquement par les éducateurs sociaux travaillant dans des homes ou foyers pour personnes en situation de handicap.

Les éducateurs sociaux observent que la conception du handicap et de son étiologie est une construction culturelle, qui dépend du contexte social et historique (Gardou, 2010).

J'ai un autre élève qui est africain, sa maman parle beaucoup de réincarnation et de dire que cet enfant c'est un Dieu, parce que, enfin, voilà, je ne sais pas exactement quelle est sa théorie, mais c'est une vision du handicap qu'on n'a pas du tout nous. (ES24, femme, 29 ans)

II ressort également des témoignages que la manière dont les membres de la famille conçoivent le handicap de l'enfant et son étiologie joue un rôle important sur leurs attitudes (rejet ou acceptation) envers la personne porteuse du handicap ainsi que leur implication dans l'intervention (Gardou, 2010; Piérart, 2013).

Des échos que j'ai eus des collègues c'est que pour la culture du Congo elle est possédée [...] c'est les mauvais esprits donc on ne va pas vers elle parce que tout d'un coup on est contaminé... (ES13, homme, 30 ans)

En ce sens, le cadre culturel donne un éclairage sur les perceptions du handicap (et de n'importe quelle autre maladie mentale ou physique) ainsi que sur le soutien apporté par la famille ou la communauté.

D'autre part, la question du normal et du pathologique sème le doute chez les éducateurs sociaux concernant les symptômes, l'étiologie ou le traitement de la maladie mentale : le comportement observé correspond-il à un 
symptôme lié au trouble psychique dont souffre la personne et doit donc être traité ou est-il juste l'expression de sa culture?

Le risque avec les troubles psychiques en tout cas c'est qu'on associe quelque chose qui serait de l'ordre du culturel à quelque chose de pathologique [...] et puis le médecin, je pense, très vite pourrait lui donner un médicament juste parce qu'il est de culture différente. C'est ça qui est terrible. (ES16, femme, 30 ans)

Tout comme le handicap, la maladie mentale est une construction culturelle: ce qui est considéré comme pathologique dans une culture peut ne pas l'être dans une autre (Paradis, 1992). Sur le plan de la santé mentale, l'approche ethnopsychiatrique invite le professionnel à comprendre et considérer la manière dont une société définit le normal et le pathologique en termes comportementaux afin de mettre en place une intervention qui soit la plus adaptée pour la personne souffrant d'un trouble psychique et sa famille (Bastide, 1968).

\section{L'éducation des enfants}

Les représentations et pratiques éducatives peuvent différer selon les points de vue des éducateurs sociaux et des parents. Concernant l'éducation en famille, des divergences ont été évoquées par les participants, notamment ceux qui travaillent dans des institutions pour enfants, adolescents et jeunes adultes en difficulté ou des structures pour parents en difficulté dans l'éducation de leurs enfants, au sujet des normes éducatives, de l'autorité parentale ainsi que du rôle et du statut de l'enfant.

Tout d'abord, les éducateurs sociaux évoquent une différence de perceptions et de pratiques dans l'éducation informelle de l'enfant, notamment au niveau de l'alimentation et du sommeil. Parfois, les professionnels considèrent même les modèles éducatifs des parents comme étant peu favorables à l'épanouissement de l'enfant.

Il y a des choses qui sont pour les enfants et il y en a d'autres qui ne sont pas pour les enfants et puis un enfant a besoin de stabilité [...] Nous, on essaie vraiment de dire à quel point l'enfant, ici en tout cas, a besoin de choses sûres pour pouvoir se développer. Le petit enfant surtout parce qu'il est sans cesse en train de se développer et s'il doit se réadapter toujours à un lieu de vie, à une personne qui le garde différente, à un lit différent, à une pièce différente, il n'a pas le temps de se développer quoi. (ES22, femme, 27 ans)

Il ressort également des témoignages que les situations liées aux représentations de l'éducation des enfants vont souvent de pair avec celles liées aux relations de genre, les pratiques éducatives des parents pouvant varier en fonction du genre de l'enfant.

La difficulté entre déjà d'avoir une fille ou d'avoir un garçon. Déjà là... Le garçon est plutôt roi, c'est viril de faire sa jeunesse, une fille elle aura nettement moins de marge de manœuvre... puis qu'on lui laisse... bon y a trop et trop peu, hein... Mais certaines elles sont vraiment enfermées, on leur offre aucune possibilité. Donc, il y a la différence du genre. (ES12, femme, 47 ans)

Ensuite, des éducateurs sociaux dénoncent des modalités de discipline très sévères, des corrections physiques, voire des châtiments corporels imposés aux enfants par les parents. Pour ces professionnels, les limites du pouvoir d'éducation des parents résultent de l'Ordonnance sur des mesures de protection des enfants et des jeunes et sur le renforcement des droits de l'enfant (2010) ainsi que du droit pénal en vigueur en Suisse.

C'est vrai que peut-être dans certains pays c'est normal de donner des gifles, de... de faire marcher à la baguette un peu son enfant, et bien là, non, ici, ce n'est pas comme ça, c'est... On est en Suisse et là... voilà quoi l'enfant... on ne frappe pas les enfants en Suisse. Et là je crois qu'ils pourront nous expliquer tout ce qu'ils veulent de leur culture, je pense que là on n'arrivera pas à accepter ça. (ES22, femme, 27 ans)

Il est important de garder à l'esprit que la maltraitance et la violence envers un enfant sont des constructions sociales, issues d'un contexte historique, culturel, moral, politique et législatif. Ainsi, certains comportements peuvent être considérés comme maltraitants ou violents dans un contexte donné, alors qu'ils sont jugés comme acceptables dans un autre, dans la mesure où ils ne s'écartent pas de l'ensemble des comportements prescrits ou permis par les valeurs morales et les normes sociales et légales du contexte (Hassan et Rousseau, 2007). 
En lien avec le rôle et le statut de l'enfant, les croyances parentales liées aux pratiques sexuelles et matrimoniales concernant les femmes sont capitales pour les familles de certaines cultures africaines : les filles sont considérées comme étant détentrices de l'honneur familial, dont le symbole est leur propre corps et, par conséquent, la conservation de la virginité (Lacoste-Dujardin, 1995; Poirier, 2000). Le mariage arrangé et la maltraitance envers les filles qui ont des relations affectives avec des pairs - " maintenant mes parents vont me dire t'es une pute » (ES9, femme, 30 ans) - sont des réalités très difficiles à vivre pour les éducateurs sociaux qui ont pour objectif de favoriser l'autonomie et l'indépendance des usagers. Ces professionnels sont parfois tentés de mettre sur pied des interventions pouvant aller à l'encontre des croyances familiales et ainsi contribuer à intensifier le conflit familial, voire à créer une rupture familiale (Manço et Freyens, 2008).

\section{Les croyances et pratiques religieuses}

Les croyances et pratiques religieuses des usagers aux références culturelles diverses (par exemple, rites ou croyances magico-religieuses) peuvent parfois susciter des réactions de surprise ou de l'incompréhension chez les éducateurs sociaux, sans qu'aucun lien avec leur milieu de pratique puisse être tissé.

Si on prie, on fait des bonnes notes et si on ne prie pas, on ne fait pas de bonnes notes. (ES8, homme, 30 ans)

J'ai été à un de ces cultes orthodoxes érythréens et c'est vrai que j'ai été assez surprise de la manière dont ça se faisait et comme ils étaient avec lui. [...] Ils mettaient les mains sur lui [...] et puis moi j'étais à côté et je comprenais rien parce que c'était en érythréen donc c'est vrai que là je me suis retrouvé assez mal à l'aise, je ne savais pas trop comment réagir et lui je voyais qu'il était aussi assez surpris. (ES3, femme, 30 ans)

Toutefois, les entretiens montrent que les éducateurs sociaux et les institutions socio-éducatives dans lesquelles ils travaillent respectent les croyances et les pratiques religieuses des usagers ainsi que les fêtes et les calendriers religieux.

Maintenant je fais référence aux cours d'éducation religieuse parce que les enfants ont tous des cours d'éducation religieuse dans le cadre de l'école. Alors effectivement pour des élèves d'origine ou de religion autre, ce n'est pas une obligation de participer. (ES19, femme, 30 ans)

\section{L'intervention et le contexte institutionnel}

Les facteurs culturels ne sont pas les seuls défis que les éducateurs sociaux rencontrent dans l'intervention en contexte multiculturel. Des éléments propres au contexte dans lequel l'intervention a lieu peuvent également en être la cause, notamment la méconnaissance de l'intervention et des mandats et missions institutionnels de la part de la famille de l'usager, ainsi qu'une compréhension différenciée des rôles et de la collaboration entre éducateurs sociaux et parents. Cette catégorie est évoquée surtout par les éducateurs sociaux qui travaillent dans des institutions pour enfants, adolescents ou jeunes adultes en difficulté.

D’une part, les éducateurs sociaux constatent que l'image que les personnes ont d'une institution (par exemple, l'école, le foyer) et de ses mandats et missions spécifiques peut changer d'une culture à l'autre.

On a plusieurs parents africains où ils ressentent que c'est une fierté finalement que leurs enfants soient éduqués par une institution [...] ils sont, du coup, relativement fiers de, voilà... c'est comme si quelque part leur enfant avait un plus par rapport aux autres parce que c'est des professionnels qui l'éduquent ou il dort chez des professionnels. (ES22, femme, 27 ans)

La méconnaissance ou à la compréhension mitigée que les familles aux références culturelles diverses ont du fonctionnement du système scolaire, social ou pénal suisse ainsi que des mandats et missions institutionnels peut être également source d'incompréhensions et de malentendus avec les éducateurs sociaux.

Mais là voilà quoi, il y avait vraiment " voleurs d'enfants, je suis son père vous n'avez absolument rien à dire là-dedans ", et je pense qu'il ne comprenait pas par exemple les systèmes de protection de la jeunesse en Suisse... il avait une 
méconnaissance complète, complète du cas... ou il refusait de la comprendre, je ne pourrais pas vous dire. (ES8, homme, 30 ans)

D'autre part, les professionnels proposent des interventions dans des formes et des rythmes qui ne sont pas toujours bien perçus et acceptés par les usagers aux références culturelles diverses et leurs proches, qui doivent comprendre et relever plusieurs défis liés à la fois aux difficultés vécues par la personne, aux facteurs culturels et aux politiques en vigueur.

Cette personne africaine qu'on a nous, nous on recherche tout le temps à l'amener chez les psychologues pour qu'elle puisse parler de son traumatisme, de tout ça, mais elle, elle a toujours dit « non, je ne veux pas, je ne veux pas ». [...] L'éducatrice qui s'occupait d'elle dans l'autre groupe m'a expliqué qu'elle a des contacts avec des personnes africaines et elles lui ont dit "Tu sais en Afrique on ne parle pas du passé ou des événements traumatisants parce que ça les fait revenir ». Ils pensent que ça va revenir s’ils parlent de ça, donc ils préfèrent oublier. (ES7, femme, 28 ans)

\section{Les trajectoires migratoires des usagers}

La dernière catégorie concerne les situations liées aux trajectoires migratoires des usagers et de leur famille. Aucun parallélisme ne peut être tiré entre cette catégorie de situation et le milieu de pratique des éducateurs sociaux.

Les éducateurs sociaux observent que la modification du contexte de vie des usagers aux références culturelles diverses peut altérer leur comportement, leur développement et leur bien-être. Les trajectoires migratoires de ces usagers et de leurs proches peuvent aussi entraver leur adaptation ou leur intégration en Suisse, ces personnes devant relever des défis spécifiques liés à la situation migratoire et s'intégrer dans un nouveau système dont elles ne connaissent pas les règles du jeu.

Les parents, souvent, s'ils viennent d'une autre culture, ils n'ont peut-être même pas le même niveau scolaire que leurs enfants, donc souvent, ils sont déjà dépassés. Donc ils sont confrontés à plein de choses qu'ils n'arrivent absolument pas à maitriser que ce soit le système, les connaissances, les connaissances qu'on pourrait appeler basiques, donc ça ils ne l'ont pas. (ES12, femme, 47 ans)

L'absence de prise en compte par les éducateurs sociaux du parcours migratoire des usagers et des transformations qui l'accompagnent peut influencer de manière négative l'intervention ainsi que la collaboration avec les proches (Vatz Laaroussi et Rachédi, 2002).

Il ressort également des témoignages que lorsque les membres de la famille de l'usager ne maîtrisent pas la langue du pays d'accueil, ils éprouvent des difficultés à connaître la situation de l'usager et l'organisation de l'institution et des services qui gravitent autour.

Il y a beaucoup de problèmes de compréhension dus à la langue ou... on ne fait pas systématiquement appel à un traducteur quand on pense que ça peut être quand même compris, mais en même temps tout n'est pas saisi de manière complètement juste, et puis des fois ça peut biaiser les choses, biaiser le message. (ES20, homme, 29 ans)

II y a une différence de langage notoire et puis depuis quelques temps en fait j'accompagne cette maman assez régulièrement aux rendez-vous médicaux de sa fille parce qu'on s'est rendus compte que bien souvent c'est difficile pour elle de nous retranscrire ce qui s'était passé. (ES19, femme, 30 ans)

Ces barrières linguistiques peuvent limiter l'accès et l'échange d'informations cruciales à propos de la situation de l'usager ou de l'organisation de l'intervention, aussi bien du côté des familles que des éducateurs sociaux (Alvarado, 2004; Piérart, 2013), voire perturber la communication et altérer l'intervention.

D'autre part, les politiques migratoires, sociales et éducatives peuvent exercer un impact sur l'accessibilité des ressources sociales, sanitaires et éducatives (Steiger et Pfiffner, 2008) ou donner lieu à des inégalités de traitement entre des usagers autochtones et des usagers ayant un permis de séjour temporaire (Chaudet et collab., 2000), comme c'est le cas dans l'exemple qui suit. 
Ce n'est pas admis de le dire, mais ce qu'il y a certainement c'est qu'il y a une manière très différente d'agir par rapport à une situation si on a des personnes qui sont avec un statut... suisse ou permis $C$ ou alors réfugiés ou... des statuts précaires on n'intervient pas parce qu'on... ou moins ou différemment. (ES11, homme, 37 ans)

Comme on peut le constater, les éducateurs sociaux sont confrontés à plusieurs défis d'ordre personnel, relationnel et contextuel dans l'intervention auprès des usagers aux références culturelles diverses et de leurs familles. Ces situations suscitent chez les professionnels des émotions, pour la plupart négatives, qui vont de la surprise et de l'incompréhension à l'impuissance et à la colère. De même, le travail avec la différence culturelle peut heurter les éducateurs sociaux dans leurs valeurs personnelles et professionnelles, les conduire à s'interroger sur la relation à l'autre, voire provoquer une remise en question de leurs cadres de référence, des représentations de la profession d'éducateur social et des pratiques professionnelles (Cohen-Emerique, 2011; Leanza, 2011; Piérart, 2013). Il peut également conduire les professionnels à s'interroger sur le contexte de l'intervention (par exemple, valeurs, mandats et missions institutionnels) ainsi que sur la société en général (par exemple, politiques migratoires, contraintes politiques et administratives). D'autre part, les témoignages montrent que les professionnels se sentent très impliqués dans l'accompagnement de ces personnes et expriment de la proximité, de l'empathie, de la confiance, voire de l'admiration pour elles. Ces situations nous renseignent également sur les ressources et les pratiques mobilisées par les éducateurs sociaux pour faire face à ces défis et à ces ressentis. Certains professionnels se sentent impuissants et restent figés dans ce questionnement, alors que d'autres développent des pratiques d'intervention adaptées aux spécificités et besoins des usagers aux références culturelles diverses, par exemple, en accordant davantage de temps, d'informations et d'explications aux familles des usagers aux références culturelles diverses, en découvrant leur cadre de références culturelles ou encore en adaptant l'intervention à leurs culture et valeurs.

Ces résultats recouvrent ce qui est connu par la littérature scientifique sur les défis que l'intervention en contexte multiculturel implique pour les professionnels de l'humain (Cohen-Emerique, 2011; Dominicé Dao et Bélanger, 2007; Edelmann, 2007; Guelamine, 2001; Layat Brun, 2007; Leanza, 2011; Piérart, 2013; Prieur, Jovelin et Blanc 2006; Steiger et Pfiffner, 2008; Verbunt, 2004). La diversité des professions (travailleurs sociaux, enseignants, médecins, etc.) et des contextes professionnels (institutions socio-éducatives, services sociaux, écoles, hôpitaux, etc.) étudiés par ces différentes études montre que le travail avec la différence culturelle peut être considéré comme un enjeu transversal.

\section{Perspectives}

Ces résultats ouvrent des pistes intéressantes pour optimiser l'intervention auprès d'usagers aux références culturelles diverses.

Au niveau de la pratique, les éducateurs sociaux sont invités à réfléchir sur leur cadre de référence personnel, leur pratique et leur propre culture professionnelle ainsi qu'à se sensibiliser à l'écoute, à la reconnaissance et à la valorisation des usagers aux références culturelles diverses. Ils sont également encouragés à contextualiser la relation d'aide, en prenant en compte les différentes dimensions subjective, relationnelle et situationnelle qui ont une influence sur eux-mêmes, sur les usagers ainsi que sur l'intervention dans son ensemble.

Au niveau de la formation en travail social, cette étude atteste de la nécessité de développer la sensibilité des éducateurs sociaux à la différence culturelle, en mettant sur pied une formation spécifique sur le savoir, le savoirêtre et le savoir-faire pour les étudiants en travail social afin qu'ils répondent de manière appropriée à la différence culturelle dans les interventions. En ce sens, la formation initiale en travail social devrait permettre l'acquisition par les futurs éducateurs sociaux des compétences interculturelles nécessaires pour l'exercice de leur profession. Autrement dit, une place assez importante devrait être réservée au développement de ces compétences psychosociales qui permettent aux futurs professionnels de faire face, plus ou moins efficacement, à des situations complexes suscitées par la rencontre avec des usagers aux références culturelles diverses dans un contexte social, politique, économique et historique inégalitaire (Manço, 2000). Dans les établissements de formation, c'est un programme didactico-expérientiel qui devrait être priorisé : une approche pédagogique qui favorise une démarche réflexive basée sur le transfert entre théorie et pratique ainsi que sur l'appropriation et l'ancrage de compétences professionnelles et personnelles par l'expérience permettant aux futurs éducateurs sociaux d'œuvrer en contexte 
multiculturel. Différents scénarios pédagogiques en lien avec cette démarche pourraient être développés autour de projets personnels ancrés dans la vie professionnelle des étudiants, de stages de formation pratique à l'étranger, de la participation à des activités facilitant les rencontres et les échanges interculturels, de jeux de rôles, de théâtres-forum, ou encore d'un e-portfolio.

\section{Références}

Abdallah-Pretceille, M. (1985). Pédagogie interculturelle : Bilan et perspectives. Dans C. Canet (dir.), L'interculturel en éducation et sciences humaines. Toulouse : Université de Toulouse Le Mirail.

Alvarado, I. (2004). Mucho camino: The experiences of two undocumented Mexican mothers participating in their child's early intervention program. American Journal of Occupational Therapy, 58(5), 521-530.

Avenir Social (2010). Code de déontologie des professionnel-le-s du travail social. Berne : Avenir Social.

Bardin, L. (2007). L'analyse de contenu (12 éd.). Paris : Presses Universitaires de France.

Bastide, R. (1968). Psychiatrie sociale et ethnologie. Dans J. Poirier (dir.), Ethnologie générale (p. 1655-1679). Paris : Gallimard.

Blanchet, A. et Gotman, A. (2006). L'entretien. Paris : Armand Colin.

Bolzman, C. (2009). Modèles de travail social en lien avec les populations migrantes : enjeux et défis pour les pratiques professionnelles. Pensée plurielle, 2(21), 41-51.

Bolzman, C. et Tabin, J.-P. (1999). Populations immigrées. Quelle insertion? Quel travail social? Genève et Lausanne : Éditions IES et EESP.

Camilleri, C. (1989). La culture et l'identité culturelle : camp notionnel et devenir. Dans C. Camilleri et M. CohenEmerique (dir.), Chocs de cultures : concepts et enjeux pratiques de l'interculturel (p. 21-76). Paris : L'Harmattan.

Chaudet, I., Regamey, C., Rosende Haver, B. et Tabin, J.-P. (2000). Migrations et travail social. Une étude des problèmes sociaux des personnes de nationalité étrangère en Suisse. Lausanne : Réalités Sociales.

Cohen, A., Rivara, F., Marcuse, E., McPhillips, H. et Davis, R. (2005). Are language barriers associated with serious medical events in hospitalized pediatric patients? Pediatrics, 116, 575-579.

Cohen-Emerique, M. (2000). L'approche interculturelle auprès des migrants. Dans G. Legault (dir.), L'intervention interculturelle (pp.161-184). Boucherville : Gaëtan Morin Éditeur.

Cohen-Emerique, M. (2011). Pour une approche interculturelle en travail social : théories et pratiques. Rennes : Presses de l’École des Hautes Études en Santé Publique.

Dominicé Dao, M. et Bélanger, E. (2007). Le patient sans diagnostic : un Autre qui dérange? Le Bulletin de l'ARIC, 45, 20-29.

Eckmann, M. (2004). De la formation à l'interculturel à l'interculturalisation de la formation. L'éducation en débats : analyse comparée, 2, 156-177.

Eckmann, M. et Bolzman, C. (2002). Quelle place pour l'interculturel dans la formation des travailleurs sociaux? Ecarts d'identité, 98, 37-41.

Eckmann, M., Sebeledi, D., Bouhadouza Von Lanthen, V. et Wicht, L. (2009). L'incident raciste au quotidien. Représentations, dilemmes et interventions des travailleurs sociaux et des enseignants. Genève : IES éditions.

Edelmann, D. (2007). Pädagogische Professionalität im transnationalen sozialen Raum. Eine qualitative Untersuchung über den Umgang von Lehrpersonen mit der migrationsbedingten Heterogenität ihrer Klassen. LIT : Wien/Zürich.

Fortin, S. et Carle, M.-E. (2007). Santé et pluralisme. Vers un nécessaire repositionnement de la culture dans l'espace clinique. Le Bulletin de l'ARIC, 45, 5-19.

Alterstice - Revue Internationale de la Recherche Interculturelle, vol. $5, n^{\circ} 2$ 
Gardou, C. (2010). Le handicap au risque des cultures. Toulouse : Erès.

Gianni, M. (2005). Politique de reconnaissance dans une société multiculturelle : enjeux et perspectives. Dans M. Eckmann et M. Fleury (dir.), Racisme(s) et citoyenneté. Un outil pour la réflexion et l'action. Genève : IES éditions.

Guelamine, F. (2001). Les faces cachées de la «différence culturelle». La construction d’une altérité ambiguë par les travailleurs sociaux. Les Cahiers du Cériem, 8, 9-22.

Hassan, G. et Rousseau, C. (2007). La protection des enfants : enjeux de l'intervention en contexte interculturel. Bulletin de l'Association pour la recherche interculturelle, 45, 37-50.

Kirmayer, L. (2011). Les politiques de l'altérité dans la rencontre clinique. L'autre, cliniques, cultures et sociétés, $12(1), 16-28$.

Lacoste-Dujardin, C. (1995). Les jeunes filles issues de l'immigration maghrébine : une problématique spécifique. France : Ministère de la justice.

Lanfranchi, A. (2002). La situation psychosociale des familles migrantes. Dans P. Wanner, R. Fibbi, M, Spescha, A. Lanfranchi, R. Caldéron-Grossenbacher et J. Krummenacher (dir.), Familles et migration. Études sur la situation des familles migrantes et recommandations de la Commission fédérale de coordination pour les questions familiales (p. 75-95). Berne : Commission fédérale de coordination pour les questions familiales.

Layat Burn, C. (2007). Représentations sociales et approche thérapeutique en milieu médical : quelles différences entre des patients de diverses cultures. Thèse en sciences de l'éducation, Université de Genève, Genève.

Leanza, Y. (2011). Exercer la pédiatrie en contexte multiculturel. Une approche complémentariste du rapport institutionnalisé à l'Autre. Chêne-Bourg : Editions Georg.

Legault, G. et Rachédi, L. (dir.) (2008). L'intervention interculturelle (2 éd.). Montréal : Gaëtan Morin Éditeur.

Loi fédérale du 24 mars 1995 sur l'égalité entre femmes et hommes (LEg) (2011). http://www.admin.ch/opc/fr/classified-compilation/19950082/index.html

Manço, A. (2000). Compétences interculturelles et stratégies identitaires. Agora débats/jeunesses, 22(1), 49-60.

Manço, A. et Freyens, F. (2008). Connaître, reconnaître et développer les ressources identitaires des jeunes filles issues de l'immigration musulmane : de la recherche à l'action scolaire préventive. Revue des sciences de l'éducation, 34(2), 399-418.

Meijer, J. (2009). Diversité multiculturelle et besoins éducatifs particuliers. Bruxelles : Agence européenne pour le développement de l'éducation des personnes ayant des besoins particuliers.

Miles, M. et Huberman, A. (2003). Analyse des données qualitatives. Bruxelles : De Boeck.

Nehring, W. (2007). Cultural Considerations for Children with Intellectual and Developmental Disabilities. Journal of pediatric Nursing, 22(2), 93-102.

OFS (2014). Étudiants des hautes écoles pédagogiques (sans HES) selon la filière d'études, la nationalité et la haute école. Consulté le 8 octobre 2015 sur : www.statistique.admin.ch.

Ogay, T. (2006). Écoles de ville et écoles de campagne, une entrée pour parler des différences culturelles avec les enseignants. Premières analyses d'une recherche longitudinale auprès d'étudiants en formation initiale d'enseignants. Formation et pratiques d'enseignants en questions. Revue des HEP de Suisse romande et du Tessin, 4, 35-53.

Ogay, T. et Edelmann, D. (2011). Penser l'interculturalité dans la formation des professionnels : I'incontournable dialectique de la différence. Dans A. Lavanchy, F. Dervin et A. Gajardo (dir.), Anthropologies de l'interculturalité. (p. 47-71). Paris : L'Harmattan.

Ordonnance du 11 juin 2010 sur des mesures de protection des enfants et des jeunes et sur le renforcement des droits de l'enfant. http://www.admin.ch/opc/fr/classified-compilation/20092333/index.html 
Paradis, A. (1992). Ethnie et folie : visages pluriels de l'anormalité. Santé mentale au Québec, 7(2), 13-34.

Piérart, G. (2013). Familles, handicap et migration. Enjeux et intervention interculturelle. Genève : IES éditions.

Poirier, M. (2000). Adolescentes maghrébines en conflit avec leur famille. Service social et médiation interculturelle. Le Sociographe, 1, 19-28.

Prieur, E., Jovelin, E. et Blanc, M. (dir.) (2006). Travail social et immigration : interculturalité et pratiques professionnelles. Paris : L’Harmattan.

Steiger, B. et Pfiffner, M. (2008). La place de l'interculturel dans les lieux de pratique du social. Lausanne : EESP.

Tatar, M. et Horenczyk, G. (2003). Diversity-related burnout among teachers. Teaching and Teacher Education, 19(4), 397-408.

Vatz Laaroussi, M. (2008). Les familles immigrantes et l'intervention intergénérationnelle. Dans G. Legault et L. Rachédi (dir.), L'intervention interculturelle ( $2^{\mathrm{e}}$ éd.) (p. 229-249). Montréal : Gaëtan Morin Éditeur.

Vatz Laaroussi, M. et Rachédi, L. (2002). Familles immigrantes des guerres en Estrie. Sherbrooke : Ministère de la Famille et de l'Enfance.

Verbunt, G. (2004). Perspectives interculturelles dans le travail social. Repères et perspectives. Paris : La Découverte. 